\title{
Inserción laboral de las (os) egresadas (os) del plan único de especializacion en enfermeria de la ENEO- UNAM: una experiencia de diez años.
}

\section{Insertion of the (old) graduates (i) the plan of speciallization in nursing only Eneo-UNAM: a ten years experience}

Dra. Lasty Balseiro Almario•, Mtra Rosa A. Zárate Grajales••, Mtra. Reyna Matus Miranda.•,

Lic. Cristina Balán Gleaves..•, Mtra. Mercedes García Cardona...., Mtro. Federico Sacristán Ruiz••

\section{Resumen}

El objetivo de este documento es analizar las características de la Inserción laboral de los egresados (as) del Plan Único de Especialización de Enfermería (PUEE) de la Escuela Nacional de Enfermería y Obstetricia (ENEO) de la UNAM en doce generaciones. La información proviene de una investigación de tipo transversal y descriptiva. Metodología . El universo lo constituyen los 1067 egresados de las generaciones de 1997 al 2008 que acepten participar en el estudio. Los datos que se reportan constituyen una tercera parte de ese universo (31.1\%). Los resultados muestran que los egresados en su mayoría laboran en el D.F. o Estado de México (85 y 7\% respectivamente). Nueve de cada diez laboran en las principales instituciones públicas del país (62\% en instituciones para población abierta y 29\% de seguridad social). El 28\% tiene la categoría de Enfermera Especialista y otro 20\% ocupa puestos de mayor responsabilidad. En cuanto a las funciones principales $68 \%$ realizan actividades que implican el trato directo con el paciente, $21 \%$ de tipo administrativo, y sólo 1.2\% participa en actividades de investigación. Discusión: las instituciones deben continuar implementando estrategias que faciliten la culminación del proceso de los estudios de posgrado ya que como se observó en este estudio quienes se han graduado tienen en mayor medida mejor posición en el empleo. Es necesaria la vinculación Universidad - Mercado laboral a fin de incorporar con mayor facilidad a los egresados en dichos escenarios. En conclusión Las instituciones educativas además de considerar las características del mercado laboral deben continuar implementando estrategias que faciliten la culminación del proceso de los estudios de posgrado ya que como se observó en este estudio quienes se han graduado tienen en mayor medida mejor posición en el empleo.

- Este artículo es derivado del proyecto PAPITT No. IN-30841-Desempeño Laboral y Desarrollo Profesional en Egresados del PUUE de la ENEO-UNAM •Profesor de Carrera Titular "B" de la ENEO, ••Profesores de Carrera Asociado "C" de la ENEO, •.•Profesor de Asignatura "B" de la ENEO, ....Profesor de Asignatura "A" de la ENEO.

Correspondencia: lastybals@hotmail.com

RECIBIDO 24-JUNIO-10

ENVIADO 30 JUNIO 10

ACeptado 2-Julio 10 


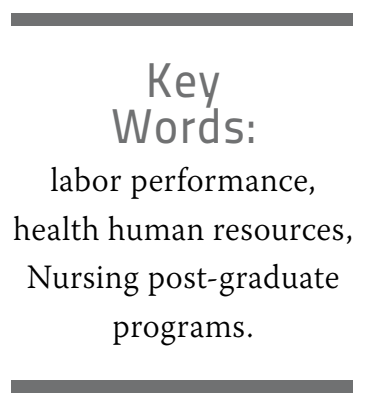

Objective: the objective of this research is to analyze the employment placing process of 12 generations from the ENEO-UnAM's Nursing Specialization Unique Plan. Methodology: basic descriptive study on a sample from the 1997-2008 generations 1,067 total graduates. Results: findings show that $85 \%$ and $7 \%$ are employed by the Federal District and State of Mexico respectively. Nine out of ten graduates are employed by the country's principal public institutions (62\% in open-population institutions and $29 \%$ in social security institutions). $28 \%$ are employed as specialist nurses, and $20 \%$ assume roles with more responsibilities. $68 \%$ perform activities related to the direct management of patients, $21 \%$ have administrative duties, and only $1.2 \%$ is fully engaged in research. Discussion: considering the results, and that graduates hold better labor positions; and in order to promote post-graduatestudies completion, education institutions should keep improving their post-graduate programs and also support their links with the shifting labor markets. Conclusion: it is necessary to strengthen the strategies which promote the synergies that contribute to the presence of the ENEO-UNAM's Nursing Specialization Unique Plan graduates in the Nursing profession and labor markets.

\section{INTRODUCCIÓN}

La inserción laboral es un término utilizado habitualmente para referirse al proceso de incorporación de los individuos a la actividad económica, también ha sido considerado un indicador con el que las sociedades occidentales miden el nivel de bienestar social de su población. Su estudio abarca las posiciones laborales ocupadas, las secuencias más recurrentes (trayectorias) así como la explicación de las diferencias observadas en estos dos aspectos. ${ }^{1}$ Es importante señalar que se habla de inserción profesional cuando se considera sólo al grupo poblacional con estudios de nivel superior, y alude al espacio que hay entre la obtención del título que legitima a una persona como profesional y el desarrollo del rol que delimita sus actividades y responsabilidades profesionales. $^{2}$

En algunos modelos de análisis se considera que en la inserción y trayectoria laboral intervienen tan- to las características del mercado de trabajo como las de los propios profesionales sin perder de vista el papel de intermediación de las instituciones educativas entre los demandantes y los oferentes de empleos u oportunidades profesionales (Instituciones y/o Empresas) ${ }^{3}$ ya que la formación académica influye de manera importante en la cualificación del denominado capital humano. Un hecho que no debe soslayarse en este análisis es el papel de la escolaridad frente al trabajo, el empleo o los ingresos, según el periodo económico de que se trate, la región geográfica, la rama de la economía e incluso de las historias y culturas de las empresas mismas. ${ }^{4}$

En el caso que nos ocupa al revisar la inserción/trayectoria de profesionales con estudios de posgrado en enfermería se debe considerar que las características generales del mercado de trabajo son en ocasiones determinantes, sin embargo, esta profesión tiene ventajas comparativas respecto a otras del área de la salud. Es decir, aunque enfermería participa de la demanda creciente de educación superior que se ha observado en el país ${ }^{1,4}$ no se ve afectada, de manera general, en la misma magnitud que otras profesiones con respecto a la insuficiencia de plazas en el mercado laboral, derivada de la crisis y la caída de la actividad económica e inclusive, de acuerdo a información que se brinda más adelante, pareciera ser que el campo de trabajo para los profesionales de enfermería con formación especializada de posgrado se está ampliando.

Desde la visión de las instituciones educativas el análisis de la inserción o posición en el mercado laboral y las características de éste permiten una primera aproximación a la relación entre formación académica y puesto que se desempeña, de tal forma que el seguimiento de egresados se considera una herramienta para evaluar la 
calidad de la formación y plantear estrategias de mejora cuyo propósito final es responder a las demandas y/o necesidades del mercado de trabajo y de la sociedad. 5,6

En el presente documento el objetivo es analizar las características que adopta la inserción/posición de los egresados del Plan Único de Especialización en Enfermería (PUEE) de la Escuela Nacional de Enfermería y Obstetricia (ENEO) correspondientes a 12 generaciones (1997-2008).

El PUEE corresponde a un proyecto de posgrado implementado en la ENEO en el año 1997 que tiene el propósito de formar especialistas de enfermería del más alto nivel, capaces de atender y resolver las diversas problemáticas que plantea la atención y el cuidado que demandan los diferentes grupos sociales y las instituciones de salud. Se considera, además, que estos estudios de especialización permiten enriquecer la formación universitaria previa, favoreciendo también el desarrollo y movilidad profesional de sus egresados. ${ }^{7}$ La ENEO, como institución rectora en este ámbito," asume que una responsabilidad inherente a todo proyecto educativo es la evaluación; por lo tanto desde la creación del PUEE se consideró la necesidad de realizar la evaluación curricular del programa (tanto interna como externa) a fin de asegurar la calidad de la formación académica que se brinda.

La evaluación interna está dirigida al programa, al rendimiento académico, al desempeño docente y al sistema tutorial. Como parte de la evaluación externa está considerado contrastar la vinculación que existe entre los objetivos del proyecto educativo y el mercado de trabajo a fin de identificar si las competencias que se favorecen en los alumnos corresponden con las requeridas en este último, así como las expectativas de los participantes sean egresados o empleadores. ${ }^{8}$ Actualmente se han realizado algunos avances en el primer senti$\mathrm{do}^{9-12} \mathrm{y}$, para abordar lo referente a la evaluación externa, se estructuró un protocolo cuyo objetivo es evaluar el desempeño y desarrollo profesional de los egresados del PUEE así como las características que asume la inserción laboral de éstos. ${ }^{13} \mathrm{El}$ presente documento se enfoca a cubrir este último aspecto.

Como ya se comentó se debe partir de que la inserción laboral está modulada por las características del mercado de trabajo; en este caso del Sistema Nacional de Salud de nuestro país, en sus Sectores Público y Privado y las Instituciones que los integran; éstas se modifican en sus características en función de las necesidades de la población y de las políticas que se establecen en los diferentes niveles de gobierno, sin perder de vista que éstas son dinámicas y heterogéneas.

Así, por ejemplo, los rasgos distintivos del sistema de salud actual en nuestro país se fueron estructurando desde principios de la década de los años cuarenta con la creación del Instituto Mexicano del Seguro Social (IMSS) de tal for-

\footnotetext{
* Es la institución con la mayor oferta de especialidades a nivel de posgrado en el país, en su inicio (1997) ofrecía tres especialidades y actualmente (2010) brinda trece. A nivel nacional únicamente en otras ocho escuelas o facultades de enfermería se ofrecen especialidades en enfermería de nivel posgrado.
}

ma que desde entonces quedaron diferenciadas dos tipos de instituciones públicas: 1) las de Seguridad Social responsables de brindar atención a la población trabajadora y sus familiares y 2) las destinadas a dar servicio a población abierta o no asegurada. Lo anterior sin olvidar a las Instituciones privadas cuyo propósito es otorgar servicios de atención a la salud a población abierta que tenga la capacidad económica para adquirirlos.

En las décadas de los 80's y los 90's las instituciones que brindan atención como parte de las prestaciones otorgadas a los trabajadores (IMSS, ISSSTE, PEMEX, MARINA, etc.) ampliaron de manera importante su infraestructura física, destacando entre ellas las que permiten brindar servicios cada vez más especializados. En el gobierno actual la creación de centros especializados ha cobrado un nuevo impulso al plantearse como parte del Plan Nacional de Desarrollo 2007-2012 la consolidación de la red de servicios de atención a la salud priorizando la red de hospitales regionales de alta especialidad y las unidades de especialidades médicas. ${ }^{15}$

Esta información permite apreciar la paulatina ampliación que ha tenido el mercado de trabajo para los profesionales de salud especializados. Sin embargo, las categorías laborales que expresan el reconocimiento que se otorga a este tipo de personal reflejan rezagos importantes, de tal forma que en algunas instituciones aún no se incluye la categoría de licenciada(o) en enfermería o bien no en todas existe el reconocimiento a la formación avanzada.

De acuerdo con los datos considerados, para el año 2001, del personal de enfermería que laboraba 
en alguna institución del sistema de salud 35\% eran auxiliares, $41 \%$ generales y $10.8 \%$ especialistas; al comparar estos datos con los reportados en 2008 se aprecia que hubo una ligera disminución en la proporción de auxiliares y se incrementa en la misma medida el de enfermeras generales; el rubro de especialistas sólo aumenta en un punto porcentual, en términos absolutos este incremento corresponde a poco más de 6300 nuevas plazas de enfermeras especialistas. ${ }^{16}$

Con base en todo lo señalado es fundamental preguntarse: ¿Cuáles son las características de la inserción laboral que están logrando los egresados del Plan Único de Especialización en Enfermería de la ENEO?

\section{METODOLOGÍA}

Los datos analizados en este documento provienen de una investigación más amplia encaminada a evaluar la inserción laboral, el desempeño y desarrollo profesional de los egresados del PUEE. ${ }^{13}$ En este documento sólo consideramos lo concerniente a las características de la inserción laboral.

Se trata de una investigación de tipo transversal y descriptiva. El universo de estudio lo integra el total de la población de egresados de las diferentes especialidades del PUEE del periodo comprendido de 1997 a 2008; los criterios de inclusión establecidos son: que pertenezcan a este universo, acepten participar en el estudio y ser egresado de las generaciones 1997-2007.

La información se recopiló por medio de una encuesta auto-administrada aplicada por la coordinación del PUEE en las unidades sedes y a través de la página web de la institución. La encuesta fue construida y validada ex profeso, se incluyeron variables sobre características generales como son: edad, sexo, especialidad y obtención de grado. Respecto a las características de la inserción laboral se consideraron: centro/institución de trabajo, categoría, antigüedad, salario $\mathrm{y}$ funciones principales.

Para la realización de esta investigación nos sujetamos a los principios de la "Declaración de Helsinki" y a los de la "Ley General de Salud", ya que se considera de riesgo mínimo de acuerdo al artículo 17 y en cumplimiento con los siguientes aspectos mencionados con el artículo 21. Se realizóanálisis descriptivo utilizando medidas de tendencia central, frecuencias y porcentajes.

\section{RESULTADOS}

El Plan Único de Especialización en Enfermería se instauró en 1997; de tres especialidades que ofrecía en su inicio actualmente ofrece trece. Los resultados presentados en este artículo corresponden a los datos recabados de las generaciones 1997-2007. Se aplicaron 332 encuestas lo que corresponde al $31.1 \%$ de la población total considereda.En la Tabla 1 se aprecia, como es característico de la profesión de

\section{Tabla 1. CARACTERÍSTICAS GENERALES}

\begin{tabular}{lll} 
& Frecuencia & $\%$ \\
Sexo & & \\
\hline Masculino & 56 & 16.9 \\
Femenino & 276 & 83.1 \\
\hline Grupos de edad & & \\
$24-34$ & 174 & 52.7 \\
\hline $35-44$ & 129 & 39.1 \\
$45-60$ & 27 & 8.2 \\
\hline Lugar de trabajo & & \\
Extranjero & 2 & 0.6 \\
\hline DF & 282 & 84.9 \\
Edo de México & 23 & 6.9 \\
\hline Otros Estados & 25 & 6.6
\end{tabular}

Tabla 2. Egresados desde 1998 en las Especialidades con mayor tiempo de operación

\begin{tabular}{lll} 
Especialidad & Egresados & $\%$ \\
\hline Cardiovascular & 123 & 13.4 \\
\hline Perinatal & 119 & 13.0 \\
Salud Pública & 102 & 11.1 \\
\hline Infantil & 237 & 25.8 \\
Adulto en Edo Crítico & 284 & 31.0 \\
\hline Anciano & 52 & 5.7 \\
Total & 917 & 100.0
\end{tabular}


enfermería, un predominio del sexo femenino; estas personas encuestadas corresponden a adultos jóvenes ya que poco más de la mitad tienen entre 24 y 34 años de edad y, en su mayoría, laboran en la zona centro del país (D.F. y Estado de México) aunque también participan de otros estados y del extranjero.

Con objeto de evaluar la demanda se consideraron únicamente las 6 especialidades que se ofrecen desde 1997 hasta el momento actual. En la tabla 2 se puede observar que de este grupo de especiali- dades las más solicitadas son las de Enfermería Infantil y de atención del Adulto en Estado Crítico, entre ambas representan más del 50\% de este total, en cambio las especialidades de cardiovascular, perinatal y salud pública tienen una proporción similar (ligeramente superior al 10\%) siendo la menos demandada la de Enfermería del Anciano.

Como puede apreciarse en la tabla 3 casi tres de cada cinco egresados laboran en instituciones públicas que proporcionan servicios para población abierta y el otro porcentaje importante es el correspondiente a quienes están insertos en instituciones de seguridad social; si sumamos estos porcentajes se tiene que 9 de cada diez egresados laboran en instituciones públicas. En consecuencia es mínima la proporción que trabaja en instituciones privadas. Otro aspecto importante de los centros de trabajo es su clasificación según nivel de atención; conforme a éste se aprecia, como era lo esperado, que las instituciones en que participan los egresados en su mayoría corres-

\section{Tabla 3. Tipo y Nivel de Atención de la Institución Laboral}

N

$\%$

Tipo de Institución

\begin{tabular}{lll}
$\begin{array}{l}\text { Servicios de salud } \\
\text { para población abierta }\end{array}$ & 206 & 62.0 \\
$\begin{array}{l}\text { Servicios de salud } \\
\text { de Seguridad Social }\end{array}$ & 95 & 28.6 \\
\hline $\begin{array}{l}\text { Servicios privados } \\
\text { de salud }\end{array}$ & 6 & 1.8 \\
Académicas & 22 & 6.6 \\
\hline $\begin{array}{l}\text { Por cuenta propia } \\
\text { e Institución otro país }\end{array}$ & 3 & 0.9 \\
Nivel de atención & 3 & 1.0 \\
\hline 1er Nivel & 67 & 21.5 \\
\hline 2o. Nivel & 214 & 68.6 \\
\hline 3er Nivel & 28 & 9.0 \\
\hline Otro & 262 & 78.9 \\
\hline Número de empleos & 70 & 21.1 \\
Uno & & \\
\hline Dos & & \\
\hline
\end{tabular}

Tabla 4. Características de la inserción laboral

\begin{tabular}{lll} 
Categoría & \multicolumn{1}{c}{ N } & \multicolumn{1}{c}{$\%$} \\
\hline Auxiliar de Enfermería & 11 & 4.4 \\
\hline Enfermera General & 145 & 45.3 \\
Licenciatura & 8 & 2.5 \\
\hline Especialista & 91 & 28.4 \\
Jefe de Servicio & 17 & 5.3 \\
\hline Supervisora o Jefe & 27 & 8.4 \\
Académicas & 18 & 5.6 \\
\hline Otros & 3 & 0.9 \\
Antigüedad & & \\
\hline 0-5 & 99 & 30.2 \\
6-10 & 100 & 30.5 \\
\hline 11-15 & 67 & 20.4 \\
16-20 & 47 & 14.3 \\
\hline 21 y más & 15 & 4.6 \\
Salario & & \\
\hline 3999 y menos & 7 & 2.2 \\
4000-8999 & 88 & 27.1 \\
\hline 9000-13999 & 147 & 45.2 \\
14000-19999 & 78 & 24.0 \\
\hline 20000 y más & 5 & 1.5 \\
Actividad principal & & \\
\hline Cuidados directos & 223 & 67.8 \\
Docencia & 29 & 8.8 \\
\hline Administración & 70 & 21.3 \\
Investigación & 4 & 1.2 \\
\hline Otra & 3 & 0.9 \\
\hline & & \\
\hline & & \\
\hline
\end{tabular}


ponden a instituciones de tercer nivel, es decir, las que por naturaleza cuentan con servicios altamente especializados $y$, en una menor proporción, a las de segundo nivel.

En cuanto al número de empleos ocho de cada diez tienen un solo empleo, pero dos de cada diez tienen dos empleos aunque este segundo porcentaje representa una cuarta parte respecto al primero no es irrelevante si se considera que la participación de las mujeres en el total de la población económicamente activa se ubica en alrededor del 40 por ciento. ${ }^{14}$ Es decir, la proporción de profesionales de enfermería que trabajan en dos lugares corresponde a la mitad del porcentaje de participación femenina total.

Las características asociadas con el empleo de los egresados (Tabla 4) permiten observar que poco más de una cuarta parte de los encuestados ha obtenido en su empleo la categoría de Especialista y otro $20 \%$ han logrado puestos de mayor responsabilidad jerárquica tanto en lo administrativo como en lo académico. La antigüedad en el empleo seguramente se vincula con la edad, por esta razón en su mayoría tienen 10 años o menos en su empleo. El reconocimiento que va obteniendo la profesión se refleja en el nivel salarial, poco más del $70 \%$ obtiene nueve mil pesos o más de ingreso salarial. Las actividades

Tabla 5. Categoría laboral según especialidad

\begin{tabular}{|c|c|c|c|c|c|c|}
\hline \multirow[t]{2}{*}{ ESPECIALIDAD } & \multicolumn{2}{|c|}{$\begin{array}{l}\text { Auxiliar, General, Li- } \\
\text { cenciada }\end{array}$} & \multicolumn{2}{|c|}{$\begin{array}{l}\text { Especialista, Jefe, Su- } \\
\text { pervisora, Académica }\end{array}$} & \multicolumn{2}{|c|}{ Total } \\
\hline & $\mathrm{N}$ & $\%$ & $\mathrm{~N}$ & $\%$ & $\mathrm{~N}$ & $\%$ \\
\hline Cardiovascular & 18 & 60.0 & 12 & 40.0 & 30 & 100.0 \\
\hline Perinatal & 9 & 34.6 & 17 & 65.4 & 26 & 100.0 \\
\hline Salud Pública & 10 & 55.6 & 8 & 44.4 & 18 & 100.0 \\
\hline Infantil & 45 & 50.0 & 45 & 50.0 & 90 & 100.0 \\
\hline Adulto EC & 59 & 59.0 & 41 & 41.0 & 100 & 100.0 \\
\hline Anciano & 2 & 20.0 & 8 & 80.0 & 10 & 100.0 \\
\hline Rehabilitación & 18 & 58.1 & 13 & 41.9 & 31 & 100.0 \\
\hline Neurológica & 1 & 14.3 & 6 & 85.7 & 7 & 100.0 \\
\hline Cultura Física y Deporte & 0 & & 2 & 100.0 & 2 & 100.0 \\
\hline Salud Mental & 2 & 33.3 & 4 & 66.7 & 6 & 100.0 \\
\hline Total & 164 & 51.3 & 156 & 48.7 & 320 & 100.0 \\
\hline
\end{tabular}

Tabla 6. Categoría laboral según titulación en la especialidad

\begin{tabular}{|c|c|c|c|c|c|c|}
\hline & \multicolumn{6}{|c|}{ Titulado } \\
\hline Categoría & \multicolumn{2}{|c|}{ SI } & \multicolumn{2}{|c|}{ NO } & \multicolumn{2}{|c|}{ Total } \\
\hline & $\mathrm{N}$ & $\%$ & $\mathrm{~N}$ & $\%$ & $\mathrm{~N}$ & $\%$ \\
\hline Auxiliar-General-Licenciada & 45 & 31.5 & 117 & 67.6 & 162 & 100.0 \\
\hline Especialista-Jefe-Supervisora-Académico & 98 & 68.5 & 56 & 32.4 & 154 & 100.0 \\
\hline Total & 143 & 100.0 & 173 & 100.0 & 316 & 100.0 \\
\hline
\end{tabular}


predominantes (68\%), como era de esperarse en profesionales de enfermería con especialidad, están relacionadas con el cuidado directo a los pacientes; alrededor del $20 \%$ reportó realizar en primer lugar actividades de tipo administrativo siendo los menores porcentajes los que corresponden a actividades de docencia e investigación.

Al evaluar el posicionamiento de los egresados en función de la categoría alcanzada apreciamos que entre las especialidades que tienen mayor tiempo de establecidas tienen mejor posición los egresados de los programas de enfermería del Adulto en Estado Crítico, del Anciano, Perinatal e Infantil y que obtener una mejor categoría laboral está vinculada a la obtención del grado académico; los datos de la tabla 6 revelan en forma clara que quienes se han graduado tienen en mayor medida mejor categoría laboral

Finalmente la relación entre año de ingreso a la especialidad y antigüedad en el empleo (no se presenta tabla) permite constatar que cerca del $80 \%$ de los egresados estudió la especialidad como una forma de mejorar su desempeño y/o buscar una promoción en el trabajo (y con ello mejorar sus percepciones económicas) pues ya estaban insertos en las instituciones de salud cuando ingresaron a la especialidad.

\section{DISCUSIÓN}

La especificidad del análisis de la inserción de quienes han realizado estudios de posgrado se centra en las posiciones alcanzadas dado que generalmente los profesionales ya han cubierto la etapa de transición entre la universidad y la incorporación a la actividad económica, la especialización o los estudios de posgrado forman parte del necesario proceso de formación avanzada a fin de acceder a mejores puestos de trabajo: éste es el caso en enfermería que se corrobora en este estudio. El propósito de mejorar de categoría o puesto no se logra siempre con facilidad ya que las instituciones de salud y educativas tienen en algunos momentos escenarios y prioridades diferentes; con frecuencia las universidades se adelantan a los hechos y el mercado laboral tarda más en construir

\begin{tabular}{|c|} 
El carácter nacional \\
de la eneo debe \\
continuar, ampliando \\
su oferta a fin \\
de contribuir a la \\
formación \\
de especialistas \\
para el país y no \\
exclusivamente en la \\
ciudad de México
\end{tabular}

las plataformas para recibir a los egresados. Además las políticas de contratación de recursos con mayor formación se encuentran en crisis desde hace varios años lo que se refleja en el número de plazas y puestos de trabajo que se ofrecen. Sin embargo, las instituciones educativas deben permanecer atentas para propiciar se culmine todo el proceso que implica la formación académica ya que, como se observó en esta investigación, es evidente que quienes han obtenido el grado de especialista en mayor medida tienen mejores puestos de trabajo.
Las variantes en la demanda de las diferentes especialidades que se ofrecen son inherentes al mayor o menor reconocimiento institucional que tiene cada una de ellas, de tal modo que ciertas especialidades cuando no encuentran espacio en los profesiogramas y en los tabuladores salariales son escasamente demandadas por las enfermeras aún sabiendo la necesidad que existe de estos profesionales para la atención de las necesidades de la población y por lo tanto su inserción laboral se ve limitada en cuanto a cambios de función y categoría laboral, por ejemplo; las especialidades de atención del anciano, salud mental y atención en el hogar.

\section{Conclusiones}

Esto conduce a reafirmar el hecho de que debe existir una relación estrecha entre la formación y las necesidades del mercado laboral, por lo que se deben hacer estudios sobre la oferta y demanda para identificar la necesidad de formación en las diferentes especialidades en particular para brindar atención a grupos específicos y/o altamente vulnerables, situación que la mayoría de los planes de estudio realizan antes de poner en marcha un nuevo proyecto; así también resulta indispensable la vinculación Universidad - Mercado laboral a fin de incorporar con mayor facilidad a los egresados en dichos escenarios.

El carácter nacional de la ENEO debe continuar, ampliando su oferta a fin de contribuir a la formación de especialistas para el país y no exclusivamente en la ciudad de México por lo que en los últimos años se han establecido sedes del PUEE en los estados de Veracruz, Oaxa- 
ca, Estado de México y Tlaxcala a fin de dar respuesta a las necesidades de formación de especialistas y a las demandas de atención de la población.

\section{RefERENCIAS Bibliográficas}

1. Salgado Vega C. Empleo y transición profesional en México. Papeles de Población, Abril-Junio 2005; 44: 255-285. Disponible en: http://redalyc.uamex.mx/ pdf/112/11204411. Consultado 24 mayo 2010.

2. Casanovas I, Escuredo B, Esteve J, Abades M, Guillaumet M, Mitjans J. Trayectoria laboral de las enfermeras de una escuela durante los 4 años siguientes a la graduación: 2000-2004. Enferm Clin. 2006; 16(5):238-43.

3. Mungaray Lagarda A. La educación superior y el mercado de trabajo profesional. Revista Electrónica de Investigación Educativa 2001; 3(1):54-66 [Consultado 27 mayo 2010] Disponible en: http://redie. uabc.mx/

4. De Ibarrola M. El incremento de la escolaridad de la PEA en México y los efectos sobre su situación laboral y sus ingresos 1992-2004. Revista Electrónica de Investigación Educativa 2009; 11(2):1-19. [Consultado 24 mayo 2010]. Disponible en: http://redie.uabc.mx/vol11no2/contenido-deibarrola.htlm.

5. Ramírez, R y Tovar MC. Egresados de las especializaciones en enfermería: Evaluación de una década.
Colombia Med. 2005; 36(supl 2): 75-86.

6. Carrascosa J, Molero D. La inserción sociolaboral de los titulados universitarios y su relación con la formación continua. REOP 2002; 13(2): 237-254.

7. Zárate Grajales RA, Balán Gleaves C. Plan Único de Especialización en Enfermería. Documento interno de la División de Estudios de Posgrado. $5^{\text {a }}$ fase, ENEO-UNAM, México, 2009.

8. Piña-Jiménez I, Morán Peña L. La evaluación curricular del Plan Único de Especialización en Enfermería. Una estrategia para garantizar su calidad. Revista Omnia Año XIII Num. 37-37 pp. 91-93. ENEO-UNAM, México 1997.

9 Piña Jiménez I. Aportaciones de los estudios de seguimiento de trayectorias académicas de alumnos, en la toma de decisiones para el mejoramiento de la calidad educativa. Documento interno de la División de Estudios de Posgrado. ENEO-UNAM 2006, Publicación electrónica.

10 Piña Jiménez I. Los estudios de trayectorias académicas y profesionales, contribuciones al estado del arte. Enfermería Universitaria. 2007; 4 (3): 32-5.

11. Piña JI, Balan GC, et al. Aprendizajes que promueve el Plan Único de Especialización en Enfermería. La perspectiva del alumno. Resultados parciales $1^{a}$ parte. Enfermería Universitaria 2008; 5 (4): 25-34.
12 Piña JI, Balan GC, et al. Aprendizajes que promueve el Plan Único de Especialización en Enfermería. Resultados parciales $2^{\mathrm{a}}$ parte. Enfermería Universitaria 2009; 6 (1): 42- 9.

13 Balseiro L, Zárate R, Matus R, Balán C, García M, Sacristán F. Desempeño laboral y desarrollo profesional de las(os) egresadas(os) del Plan Único de Especialización en Enfermería de la ENEO. Documento interno de la División de Estudios de Posgrado. Proyecto aprobado Registro 042; ENEOUNAM. PAPPIT IN308410.

14 INEGI. Banco de Información Económica. ENEU 2004. [Consultado 22 de mayo 2010]. Disponible en: http://dgcnesyp.inegi.org.mx/cgiwin/bdieintsi.exe/SER65262

15 Camarillo Ruíz MN. La formación de enfermería en el IMSS. Rev Enferm IMSS 2001; 9(3): 125-126.

16 Plan Nacional de desarrollo 2007 - 2012, 3.2 Salud. [Consultado 27 julio 2010] Disponible en: http:// pnd.calderon.presidencia.gob. mx/igualdad-de-oportunidades/ salud.html

17 Dirección General de Información en salud (DGIS). Base de datos de recursos (Infraestructura, material y humanos) 2001-2008. [en línea]: Sistema Nacional de Información en Salud (SINAIS), [México): Secretaría de Salud, [consultado 13 de junio 2010]. Disponible en:http://www.sinais.salud.gob. mx. 